\title{
Turning Ability in Stroke Survivors: A Review of Literature
}

\author{
Haidzir Manaf, ${ }^{1}$ Maria Justine, ${ }^{1}$ Mazlifah Omar, ${ }^{2}$ \\ Khairil Anuar Md Isa, ${ }^{3}$ and Zoolfaiz Salleh ${ }^{1}$ \\ ${ }^{1}$ Physiotherapy Department, Faculty of Health Sciences, Universiti Teknologi MARA (UiTM), Puncak Alam Campus, \\ 42300 Puncak Alam, Selangor, Malaysia \\ ${ }^{2}$ Rehabilitation Medicine Department, Faculty of Medicine, Universiti Teknologi MARA (UiTM), Sungai Buloh Campus, \\ 47000 Sungai Buloh, Selangor, Malaysia \\ ${ }^{3}$ Basic Science Department, Faculty of Health Sciences, Universiti Teknologi MARA (UiTM), Puncak Alam Campus, \\ 42300 Puncak Alam, Selangor, Malaysia
}

Correspondence should be addressed to Haidzir Manaf, haidzirmanaf@yahoo.com

Received 28 June 2012; Accepted 2 August 2012

Academic Editors: C.-L. Kao, J. L. Leasure, K.-H. Pantke, and H. Unalan

Copyright (C) 2012 Haidzir Manaf et al. This is an open access article distributed under the Creative Commons Attribution License, which permits unrestricted use, distribution, and reproduction in any medium, provided the original work is properly cited.

\begin{abstract}
The aim of this paper is to explore the research literature on the turning ability among stroke survivors. Stroke is one of the top five leading causes of death and disability in Malaysia. Stroke survivors reported a higher rate of fall incidences with turning while walking has been the major contributor in most of the incidences. The attentional task requires stroke survivors to have higher cognitive and attention function, and sound muscle coordination in order to perform multitask activities such as driving, walking and turning while talking, carrying an object (holding a plastic bag), navigating corners and overcoming obstacles within the base of support. Most of the previous studies have focused on the kinematic and gait parameters measurement of turning ability among stroke survivors. However, studies conducted on muscle activity using electromyography to evaluate the time pattern of muscle contraction during turning event is lacking. With regards to dual-task ability among stroke survivors, there is insufficient information or research into dual task (motor and cognitive) ability during turning. Further studies are needed to understand the effects of dual-task activity on muscle activity and gait parameters and how this impairment affects the turning ability. This needs to be addressed in order to prevent falls among stroke survivors.
\end{abstract}

\section{Introduction}

Stroke or cerebrovascular accident is the second most common cause of death and disability worldwide [1]. In Malaysia, epidemiological data in 2002 showed that stroke was one of the top five leading causes of death with a mortality rate of 11.9 per 100,000 population [2]. In addition, the National Stroke Association of Malaysia highlighted that around 40,000 Malaysian are disabled due to stroke every year [3]. This indicates that the high number of stroke incidences may increase demand of health services as well as increases burden to caregivers.

Stroke is defined as the "rapidly developing signs of focal (or global) disturbance of cerebral function, which is lasting more than 24 hours (unless interrupted by surgery or death), with no apparent nonvascular cause" [4]. In other words, stroke is a disease caused by the disruption of blood vessels in the brain, such as ischemic and hemorrhagic [5].
Consequently, those who survived are presented with significant impairment that may affect sensorimotor, swallowing, perception, vision, emotion, cognitive, and communication which in turn may predispose them to various complications and long-term disability [6]. Fall seems to be the most common reported complication especially among chronic survivors. Epidemiological studies have reported that the incidences of falls range from $16 \%$ to as high as $73 \%$ after 6 months of stroke [7-9] with at least $20 \%$ reported multiple falls [10]. Moreover, falls occur frequently during walking [11].

In 2001, World Health Organization (WHO) introduced the International Classification of Functioning, Disability and Health (ICF) as the model to explain in a standardized language regarding human function in the context of health and health related issues [12]. The ICF consists of the following components: body functions and structures, activity, participation, and environmental and personal factors. The 
ICF can also be used for classifying the outcome measures and physiotherapy management [13]. As such, this paper is organized based on the concept suggested by the ICF model, as shown in Figure 1.

Based on the ICF model, neurological deficits from stroke are segregated into impairments of the body function, activity limitations and participation restrictions [12]. The body function affected in stroke including muscle weakness, alteration in muscle tone, muscle contraction, sensation, speech, balance as well as feeding, which can be defined as problems arising from physiological loss.

Activity can be defined as the execution of a task such as walking, climbing stairs, eating, and toileting while participation is the involvement in work, leisure, and social activities. One of the common activity limitations among stroke survivors is walking dysfunction. Limitation of the walking potential causes most of the stroke survivors to be bedridden, reduce cardiovascular activity, less participation in the community, and some may end up with psychological disturbance [15].

The purpose of this article is twofold. First, it explains the impairment and activity limitation following stroke mainly focusing on the sensorimotor function and balance performance. Indeed both domains have significant implication on the mobility of stroke survivors especially when performing daily functions that require substantial walking coordination. Second, it discusses the characteristics of walking and turning in stroke survivors and compares this with those of the normal or healthy adults.

\section{Impairments and Activity Limitations in Stroke Survivors}

2.1. Sensorimotor Functions. Within the body function and structure component of the ICF, sensorimotor deficits are considered to be the main causes of physical and activity limitation in stroke survivors [16]. In the early stage of stroke, patients may experience impairment of motor function such as flaccid paralysis and weakness of the upper and lower limbs [17], loss of proprioception and sensation [18]. Gradually, this condition may be replaced by the development of spasticity, hyperreflexia, and abnormal mass movement pattern. With regards to the level of severity, a stroke patient can be described as either hemiplegia or hemiparesis.

Spasticity becomes one of the vital causes of disability among poststroke patients due to functional limitations during performance of activities of daily living. It has been reported that the occurrence of spasticity is about $20 \%$ in stroke survivors [19]. Spasticity is the impairment of the sensorimotor functions characterized by an increment in muscle tone with exaggerated tendon jerks, resulting from hyper excitability of the stretch reflex. In most cases, sign of spasticity may be seen in certain groups of muscles such as elbow flexors, wrist flexors, and quadriceps and calf muscles. In addition, spasticity of the affected ankle plantarflexors also affects gait symmetry in stroke survivors [20]. Previously, the treatment methods in stroke rehabilitation were

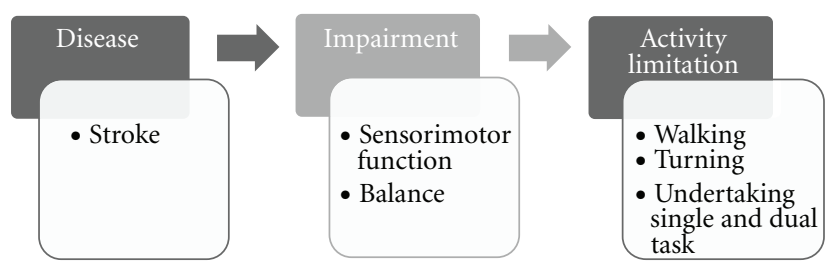

FIGURE 1: Conceptual framework showing impairments and activity limitations in stroke survivor according to the ICF core sets for stroke [14].

mainly aimed at stimulating flaccid muscles and inhibiting spasticity. However, the current trend is changing towards improving muscle strength and increasing participation in physical activity [21].

The relationship between muscle strength and walking activity has been investigated [22]. It was revealed that motor function is the dominating predictor towards independence in self-care as compared to higher-order perception, lower-order perception and orientation [23]. According to Desrosiers et al. [24], motor recovery occurs at a different time in the upper and lower extremities, with recovery of upper extremity going on in a while and extends into the stage after being discharged from active rehabilitation. In contrast, stroke survivors may regain function of the lower limb earlier than the upper limb possibly due to the focus of the rehabilitation that usually begins with the lower limb. This is supported by previous study that lower extremity was a significant predictor for gait endurance and functional balance [25].

Recovery towards walking ability is essential for stroke patients to be independent at home and in the community. As such, early rehabilitation training may restore and enhance the lives of stroke survivors. Stroke survivors may undergo a form of functional training in which it helps the patient to improve abilities such as bed mobility, sit-tostand, walking, and activity of daily living (ADL). A study carried out in Malaysia reported that $82 \%$ of stroke survivors showed improvement in most of the functions (both motor and cognitive) with $60 \%$ of them were independent in ambulation and $40 \%$ required assistance after 3 months post stroke [26]. However, this study did not mention whether these stroke survivors attended rehabilitation. Therefore, understanding and performing early assessment of motor function among stroke survivors are crucial to ensure the success of rehabilitation training.

2.2. Balance Performance in Stroke Survivors. The loss of ability to control postural balance is another crucial deficit following stroke. According to O'Sullivan and Schmitz [27], postural balance is a complex interaction of sensory, motor, and central nervous system. Inability to stabilize the body within the center of gravity happens particularly during dynamic walking and during changing direction such as preplanned or sudden turning. Impaired postural control also influences other daily life activities such as overcoming obstacles [28], reaching activity [29], and regaining stability following external perturbations [30]. 
There is a high risk of falls during functional activities among stroke survivors. Performance-based test of balance was reported as being a significant predictor for falling [31]. Patients who experienced at least one fall showed significantly lower scores on Berg Balance Scale (BBS) [32]. During the early stage of stroke, fall may occur mainly during indoor activities while among community-dwelling chronic stroke survivors fall occurs mainly during walking [11].

2.3. Gait Pattern in Stroke Survivors. The abnormality of gait in stroke survivors can be compared with normal gait based on gait kinematic, parameters, and muscle activity. Several studies have clearly demonstrated that gait velocity, cadence, stride length, and stride symmetry were important parameters for the gait analysis [33-36]. During walking, task requirements include sequences of limb motion that propel the body forward, while maintaining stability. The head, neck, and trunk are actively maintaining neutral vertebral alignment, and both arms swing in the directions opposite to those of the ipsilateral legs. Therefore, walking task requires bilateral, symmetrical, and coordinated movements of all limbs and posture.

In stroke survivors, extensor synergy causes interruption to the initiation and forward propulsion of hip flexion during the swing phase that may lead to inadequate ground clearance [37]. In the absence of forward rotation of the pelvis and hip flexion, the affected lower limb is brought forward by the circumduction pattern. Another factor is due to the increase tone in the adductor muscle that is causing the affected lower limbs to cross over to the nonaffected limb. This has also been noted to interfere grossly with the forward propulsion.

In a healthy population, the ankle is in $90^{\circ}$ flex once the weight is transmitted to the fore foot during heel strike. Later the sole is gradually lowered, which is controlled by the dorsiflexor (eccentric), to prevent slapping on the floor. In contrast, there is a lack of ankle dorsiflexion during the early stance phase in the stroke survivors. The affected limb comes down on the entire sole, or toes during heel strike. If there is a pronounced inversion, there is even more weight bearing on the lateral aspect of the foot. During midstance, spasticity of the calf muscle is preventing the forward shift of body weight leading to the lack of push off and force that is required to initiate a swing.

In another study, Hall et al. [38] found that both paretic soleus and nonparetic quadriceps were the fundamental determinants of walking speed in stroke survivors. Soleus is also a significant contributor to forward propulsion during preswing phase $[38,39]$. Therefore, an increment in the motor control of the hip flexors and ankle plantarflexors in the paretic side, and reduced hip and knee extensor in the nonparetic side is crucial to accomplish higher functional walking status.

2.4. Gait Training in Stroke Survivors. Independent in walking is one of the rehabilitation goals for stroke survivors. Physiotherapy plays an important role during rehabilitation stages to improve the mechanics and quality of walking.
Conventionally, gait training concepts were focused on restoring motor control of the paretic limb, postural control, and using assistive device such as quad cane. However, prolonged use of these devices can cause compensation techniques which increase asymmetrical weight bearing and the workload of unaffected limbs. Therefore, physiotherapist continues restoration of the normal movements of the trunk, pelvis, and lower extremity while walking and improving symmetry and weight bearing on the paretic side [40].

Recently, repetitive training of tasks results in improvement of lower limbs functions and walking performance [41]. Previous study revealed the effectiveness of repetitive task training conducted in circuit class training which includes the following: (1) graded strengthening using functional tasks (e.g., repetitive rising from a chair), (2) aerobic training (e.g., cycle ergometry), and (3) a battery of walking tasks with challenging postural control demands (e.g., stepping over obstacles) [42]. Other examples of specific tasks are moving from sitting to standing, standing balance and weight-shifting activities, stepping forward and backward and forward progression.

Turning task is also an important component during walking in stroke survivors. However, there is insufficient knowledge regarding turning task as the component in repetitive task gait training. In the next section, the mechanism involved in turning is further explained, in relation to turning strategies, muscle activity, balance performance, and attention capacity.

\section{Turning in Stroke Survivors}

Nearly every functional task performed during the day requires some amount of turning. In the home setting, turning may happen spontaneously during everyday activities such as making a cup of tea or taking food out of the refrigerator and bringing it to the dinner table. Meanwhile in the community, walking and turning are essential activities that enable a person to coordinate locomotion towards the desired location and overcome obstacles. Turning activity in healthy young individuals during walking is indeed a complex task that requires the integration of muscle coordination, visual, vestibular, and proprioceptive information [43] in sequence with horizontal reorientation of gaze, head, and body segments [44]. Other than that, turning activity also requires postural adjustment [45], reorientation of axial body segments [46], and modification of the gait parameters [47].

Falls associated with turning is common in stroke survivors [48] especially among community-dwelling, chronic survivors [49]. Previous study showed that fall injuries during turning tasks are eight times more common than during walking straight forward [50], and the incidence of falls in stroke survivors is more frequent toward the paretic side [51]. Following falls, stroke survivors may suffer from soft tissue injuries, fractures, depression, and inactivity due to fear of falling [9]. Indicators of difficulty of turning were based on 4 categories of movement during turning: (1) staggering during the turn, (2) the absence of pivoting 
during the turn, (3) using 5 or more steps or weight shifts to accomplish the turn, and (4) taking 3 seconds or longer to accomplish the turn [52]. Therefore, it is paramount to understand the strategies for turning among this physically challenged population in order to identify what rehabilitation strategies should be.

3.1. Turning Strategies. Established strategies to control turning are crucial for safe and better postural adjustment during changing direction. It involves slowing down gait speed or stopping action before rotation of head, trunk, and lower limb towards the new direction. In a healthy population, the sequences of walking and turning demonstrated in order with horizontal reorientation of visual, head, and body segments (trunk) in the direction of the turn $[44,46]$.

Patla et al. [46] categorized turning into two types: step and spin (pivot) turning. Step turning involves a change in the direction to the opposite side of the stance limb, whereas a spin turn involves a change in the direction towards the stance limb. Studies have compared advantages between both strategies. Hase and Stein [53] suggested that step turn strategy is convenient and more stable because it involves a wider base of support while changing direction as compared to spin turn in a healthy subject. This was supported by Taylor, Dabnichki, and Strike [54] who compared $90^{\circ}$ spin and step turns on three-dimensional kinematic and kinetic analysis among the healthy population. They found that step turn has more advantages than the spin turns such as a wider base of support and less involvement of movement coordination.

Kinematics in turning has also been examined in stroke survivors. It was concluded that the ability to change walking direction such as turning is impaired in chronic stroke survivors [55] that may put them at risk of falls and limitations in ADL. Other studies agreed that dysfunction of the reorientation of axial body segments towards the new direction is a major deficit in stroke survivors $[43,56]$.

It is also possible that stroke survivors show poorer performance in the turning activity as compared to healthy subjects. This was supported by previous study which examined the differences of kinematic and turning coordination between stroke survivors with and without a history of falling, and healthy age-matched subjects during the $180^{\circ}$ turning [56]. The subjects performed Timed "Up \& Go" (TUG) test with a video recording of full-body kinematics. Time and number of steps to turn as well as measurement of axial segment coordination were taken. The result of the study revealed that stroke survivors especially with falls history took significantly longer time to turn compared to the control subjects. However, there were no differences of kinematic and axial segment coordination found in the performance during turning that might contribute to risk of falls. As such, further investigation is timely to identify if other factors such as cognitive, attention or psychological status do affect turning strategies among stroke survivors.

3.2. Muscle Activity during Turning. The research to date has tended the focus on muscle activity among healthy population and Parkinson disease. Hase and Stein [53] evaluated the muscle activity in turning during walking among the healthy population. They found that deceleration mechanism requires muscle activity from distal to proximal one side of the body (soleus, biceps femoris, and erector spinae). The activities of gluteus medius (elevation of the pelvis) and ankle inverters may contribute to the control of trunk rotation. This activity is related to the timing of the opposite foot strike and the step cycle when the stimulus was applied. Turning activity is also completed without the rearrangement of the original walking rhythm.

So far no related studies were found in stroke survivors; however, muscle activity during turning has been conducted among Parkinson disease patients. Complication of Parkinson disease results in turning difficulty that can lead to fall incidences. Hong et al. [57] analyzed the kinematics and muscle activity of subjects with Parkinson disease and control as they turned 180 degrees. There was no significant difference in the overall muscle activity patterns for both groups. The researchers suggested further investigation that can reveal turning difficulty at home and in the community environment. It may include activities of a motor task (holding an object) or cognitive task (talking, calculating) while performing turning.

Although studies have been carried out on muscle activity during turning in healthy and Parkinson disease, no single study exists which adequately covers stroke survivors. It is important for future research to investigate the muscle activity especially on time pattern and maximum voluntary contraction during turning $180^{\circ}$ in community stroke survivors.

3.3. Gait Parameters during Turning. The process of turning consists of slowing walking speed, rotating the body, and changing steps direction. Measurement of gait in turning is using temporal-spatial parameters such as the number of steps, time, gait velocity, step width, and step and stride length. Thigpen et al. [52] reported that young-healthy adults used a spin (pivot) strategy and completed the turn in less than 2.5 seconds with an average of 2 steps. Meanwhile, most of older adults with turning difficulty used multiple steps (5 or more), showed marked hesitancy, pauses, and stops during turning. On the other hand, stroke survivors showed a reduction in gait parameters during turning $180^{\circ}$. They required a longer time, increased number of steps and staggered when turning $[52,56,58-61]$.

3.4. Balance Control during Turning. Maintaining balance within the base of support is a complex functional motor task. Winter [62] defined balance as the interaction of body posture to prevent falling and is associated with inertial forces acting on the body segments. In fact, the balance instability during walking can be attributed to the reduce range of motion, muscle strength, motor coordination, sensory integration, and abnormal muscle tone and cognition.

Stability on different bases of support at home and in the community walking is required to navigate corners and overcome obstacles. Segal et al. [63] in their comparative 
study found that local dynamic stability and lower limbs kinematic (stride and limb length) [47] during turning were decreased as compared to straight walking. In another study, Strike and Taylor [64] found that ground reaction forces of anterior-posterior and medial-lateral impulses were significantly greater in turning than straight walking. The study indicated increase support during the process of medial transfer, slow down and forward motion. However, they only measured the $90^{\circ}$ turning only. It would be more beneficial if they measured the $180^{\circ}$ turning as well.

Performance-based outcome measure can be used to measure turning difficulty. A previous study has found that stroke survivors who presented with difficulty in turning require more than four seconds to turn $360^{\circ}$ based on the BBS test [61]. Another study reported that Timed Up and Go (TUG) test is an indicator of difficulty when turning if it requires longer than three seconds.

Fear of fall seems to be common among stroke survivors, due to the inability to control balance. Faria et al. [59] reported that fear of falling might contribute to falls among stroke survivors other than motor impairment. This was based on the study that compared $180^{\circ}$ turning ability between stroke patient and control group. Both groups performed turning based on the Timed "Up and Go" test (TUG) for both directions. The results of the study showed that the time to complete the test were slower in stroke survivors than the control subjects. The time to complete TUG was found to be significantly correlated with gait speed, balance, and fear of falling.

3.5. Cognitive Function during Turning. Many activities of daily living require a person to complete multitasks concurrently. Normally, healthy populations are able to execute cognitive and motor tasks simultaneously regardless of the speed and direction. The complexity and familiarity of the task determine the degree of attention that is required. Attention is the information processing capacity of the individual to focus on a task [65]. In stroke survivors, attention deficits are categorized into sustained and divided attention. Divided attention can be determined by asking subjects to perform the task simultaneously [27].

There are three main theoretical models of dual-task interference in stroke survivors: (1) the capacity-sharing (2) the bottleneck, and (3) the cross-talk model [66]. In the capacity-sharing model, the information processing capacity is divided into 2 portions when stroke survivors performed 2 tasks simultaneously. Once information processing capacity is exceeded, dual-task interference occurred and resulting in declination of performance on one or both of the tasks. Meanwhile in the bottleneck model, 2 similar tasks performed simultaneously causing interference because both tasks compete for the use of the same pathway. On the other hand, the cross-talk model assumed that 2 similar tasks will reduce interference, because the use of the same pathway reduces the traffic of information processing capacity.

A few studies have been published on the effects of cognitive tasks on balance and stability in stroke survivors. Bensoussan et al. [67] examined the effects of an attention task on postural control performances between stroke survivors and a control group. Measurement of sway was taken during 30 seconds in standing with eyes open, standing with eyes open while performing a basic arithmetic task and standing with eyes close. The result of the study revealed that dual cognitive task (arithmetic and eyes closed task) reduces the postural performances of stroke survivors. Another study investigated the dual-task balance ability among fifty-four stroke survivors at 6 and 12 months post-discharge from hospital. Postural sway was examined during standing on a force plate with feet together and eyes closed. Sway in standing while performing a cognitive (shopping list) task and without cognitive task was assessed. The result of this study showed that stroke fallers swayed more than non-fallers [68].

Numerous studies have reported the interference of dual motor and cognitive task on walking performance. In a study done by O'Shea, Morris, and Iansek [69], participants with Parkinson Disease were analyzed using a clinical stride analyzer when performing dual motor (coin transference) and dual cognitive task (subtractions by threes) in a 14-meter length of straight walking. The result showed an increment in a double-limb stance as well as reduction in stride length, cadence, and gait speed. Yang et al. [70] identified that community stroke survivors have difficulty performing two motor tasks concurrently. Their study demonstrated reductions of gait performance between the community stroke survivors and control subjects during buttoning and tray-carrying task. In addition, Melzer et al. [71] compared the voluntary step behavior between chronic stroke survivors and sex-matched controls during single and dual-task conditions. Both groups performed forward and backward rapid voluntary stepping under two conditions: awaiting a cutaneous cue (single task) and awaiting a cutaneous cue while performing an attention-demanding task (reading out loud the color of the ink of the projected color name). Step initiation, preparatory and swing phases, foot-off time, and foot-contact time were extracted from the center of pressure and ground reaction data. For both conditions (single and dual task), chronic stroke survivors were significantly slower than healthy controls in all step parameters. The foot-contact time increased in dual-task conditions compared to a single task for both groups.

Bowen et al. [72] investigated whether performing cognitive tasks while walking might affect stroke survivors' balance and gait velocity. They performed two tasks, walking only and walking with concurrent cognitive activity (verbal). The result of the study revealed that there was a reduction of balance and gait velocity while performing a verbal, cognitive task in stroke survivors. Other researchers also investigated the relationship between gait and three different cognitive tasks in stroke survivors. Gait parameters (gait speed, stride time, and cadence) were more affected by speech task compared to memory and auditory task while walking [73].

Turning ability requires higher cognitive and attention function with sound muscle coordination in order to support the body within the base of support. Impaired ability to sustain attention and coordinate muscle simultaneously may contribute to falls incidence. More recently, considerable studies have been carried out into dual-task activity during straight walking among stroke survivors [70, 72-74]. 
However, little is known about the effects of dual task in gait performance during turning event. The introduction of secondary task through dual tasking (reaching for objects, carrying an object, talking) may reveal important information about cognitive function and attention demands during turning activity.

\section{Summary and Conclusion}

The literature review highlighted the needs for further research into this condition especially as it affects most of the stroke survivors. Stroke survivors may have difficulty performing turning task especially in the community. This is because turning requires higher cognitive and attention function when combined with multitask activities such as walking and turning while talking, carrying an object (holding a plastic bag), navigating corners, and overcoming obstacles.

Most previous studies focused on the kinematic and gait parameters measurement of turning ability among stroke survivors. However, there is a lack of study focusing on muscle activity using electromyography to evaluate maximum voluntary contraction and time pattern during turning event. With regards to dual-task ability among stroke survivors, there is insufficient information or research into dual motor task ability during turning event. This needs to be addressed in order to prevent falls among stroke survivors. This is because turning activity combined with other motor tasks such as holding a cup demands sound cognitive and muscle activity in order to perform it safely.

There are gaps which need to be addressed in order to improve the effectiveness of gait rehabilitation during community walking. Therefore, it is crucial for further study to evaluate the effects of dual tasking that might affect muscle activity and gait parameters while performing $180^{\circ}$ turning.

\section{Conflict of Interests}

None of the authors have conflict of interests to disclose.

\section{Acknowledgment}

This study was supported by Research Management Institute (RMI) via Research Intensive Faculty (RIF) grants, Universiti Teknologi MARA (UiTM) Malaysia.

\section{References}

[1] C. J. L. Murray and A. D. Lopez, "Mortality by cause for eight regions of the world: global burden of disease study," The Lancet, vol. 349, no. 9061, pp. 1269-1276, 1997.

[2] Ministry of Health, "Clinical Practice Guidelines: Management of Ischaemic Stroke," 2006, http://www.moh.gov.my/ cpgs? offset $=20$.

[3] NASAM, 2011, http://www.nasam.org/english/preventionwhat_is_a_stroke.php.

[4] P. Thorvaldsen, K. Kuulasmaa, A. M. Rajakangas, D. Rastenyte, C. Sarti, and L. Wilhelmsen, "Stroke trends in the WHO MONICA project," Stroke, vol. 28, no. 3, pp. 500-506, 1997.
[5] C. R. Mohamed, The educational needs of home-based stroke patients and family caregivers in Malaysia [Ph.D. thesis], Victoria University of Wellington, 2010.

[6] H. M. Dewey, L. J. Sherry, and J. M. Collier, "Stroke rehabilitation 2007: What should it be?" International Journal of Stroke, vol. 2, no. 3, pp. 191-200, 2007.

[7] A. Czernuszenko and A. Czlonkowska, "Risk factors for falls in stroke patients during inpatient rehabilitation," Clinical Rehabilitation, vol. 23, no. 2, pp. 176-188, 2009.

[8] L. Nyberg and Y. Gustafson, "Patient falls in stroke rehabilitation: a challenge to rehabilitation strategies," Stroke, vol. 26, no. 5, pp. 838-842, 1995.

[9] A. Forster and J. Young, "Incidence and consequences of falls due to stroke: a systematic inquiry," British Medical Journal, vol. 311, no. 6997, pp. 83-86, 1995.

[10] B. Belgen, M. Beninato, P. E. Sullivan, and K. Narielwalla, "The association of balance capacity and falls self-efficacy with history of falling in community-dwelling people with chronic stroke," Archives of Physical Medicine and Rehabilitation, vol. 87, no. 4, pp. 554-561, 2006.

[11] J. E. Harris, J. J. Eng, D. S. Marigold, C. D. Tokuno, and C. L. Louis, "Relationship of balance and mobility to fall incidence in people with chronic stroke," Physical Therapy, vol. 85, no. 2, pp. 150-158, 2005.

[12] I. Lemberg, I. Kirchberger, G. Stucki, and A. Cieza, "The ICF core set for stroke from the perspective of physicians: a worldwide validation study using the delphi technique," European Journal of Physical and Rehabilitation Medicine, vol. 46, no. 3, pp. 377-388, 2010.

[13] S. Mudge and N. S. Stott, "Outcome measures to assess walking ability following stroke: a systematic review of the literature," Physiotherapy, vol. 93, no. 3, pp. 189-200, 2007.

[14] S. Geyh, A. Cieza, J. Schouten et al., "ICF core sets for stroke," Journal of Rehabilitation Medicine, vol. 36, supplement 44, pp. 135-141, 2004.

[15] M. T. Glamcevski and J. Pierson, "Prevalence of and factors associated with poststroke depression: a Malaysian study," Journal of Stroke and Cerebrovascular Diseases, vol. 14, no. 4, pp. 157-161, 2005.

[16] L. Ada, S. Dorsch, and C. G. Canning, "Strengthening interventions increase strength and improve activity after stroke: a systematic review," The Australian Journal of Physiotherapy, vol. 52, no. 4, pp. 241-248, 2006.

[17] R. W. Bohannon and A. W. Andrews, "Relationships between impairments in strength of limb muscle actions following stroke," Perceptual and Motor Skills, vol. 87, no. 3, part 2, pp. 1327-1330, 1998.

[18] D. L. Smith, A. J. Akhtar, and W. M. Garraway, "Proprioception and spatial neglect after stroke," Age and Ageing, vol. 12, no. 1, pp. 63-69, 1983.

[19] D. K. Sommerfeld, E. U. B. Eek, A. K. Svensson, L. W. Holmqvist, and M. H. von Arbin, "Spasticity after stroke: its occurrence and association with motor impairments and activity limitations," Stroke, vol. 35, no. 1, pp. 134-139, 2004.

[20] P. Y. Lin, Y. R. Yang, S. J. Cheng, and R. Y. Wang, "The relation between ankle impairments and gait velocity and symmetry in people with stroke," Archives of Physical Medicine and Rehabilitation, vol. 87, no. 4, pp. 562-568, 2006.

[21] R. P. S. van Peppen, G. Kwakkel, S. Wood-Dauphinee, H. J. M. Hendriks, P. J. van der Wees, and J. Dekker, "The impact of physical therapy on functional outcomes after stroke: What's the evidence?" Clinical Rehabilitation, vol. 18, no. 8, pp. 833862, 2004. 
[22] U. B. Flansbjer, D. Downham, and J. Lexell, "Knee muscle strength, gait performance, and perceived participation after stroke," Archives of Physical Medicine and Rehabilitation, vol. 87, no. 7, pp. 974-980, 2006.

[23] B. Bernspang, K. Asplund, S. Eriksson, and A. R. Fugl-Meyer, "Motor and perceptual impairments in acute stroke patients: effects on self-care ability," Stroke, vol. 18, no. 6, pp. 10811086, 1987.

[24] J. Desrosiers, F. Malouin, C. Richards, D. Bourbonnais, A. Rochette, and G. Bravo, "Comparison of changes in upper and lower extremity impairments and disabilities after stroke," International Journal of Rehabilitation Research, vol. 26, no. 2, pp. 109-116, 2003.

[25] P. Kluding and B. Gajewski, "Lower-extremity strength differences predict activity limitations in people with chronic stroke," Physical Therapy, vol. 89, no. 1, pp. 73-81, 2009.

[26] B. A. R. Rameezan and O. Zaliha, "Functional status of acute stroke patients in University Malaya Medical Centre (UMMC), Kuala Lumpur, Malaysia," Medical Journal of Malaysia, vol. 60, no. 5, pp. 548-559, 2005.

[27] S. B. O'Sullivan and T. J. Schmitz, Eds., Physical Rehabilitation, F.A. Davis Company, Philadelphia, Pa, USA, 2007.

[28] D. M. Cameron, R. W. Bohannon, G. E. Garrett, S. V. Owen, and D. A. Cameron, "Physical impairments related to kinetic energy during sit-to-stand and curb-climbing following stroke," Clinical Biomechanics, vol. 18, no. 4, pp. 332-340, 2003.

[29] A. Kusoffsky, I. Apel, and H. Hirschfeld, "Reaching-liftingplacing task during standing after stroke: coordination among ground forces, ankle muscle activity, and hand movement," Archives of Physical Medicine and Rehabilitation, vol. 82, no. 5, pp. 650-660, 2001.

[30] R. R. Holt, D. Simpson, J. R. Jenner, S. G. B. Kirker, and A. M. Wing, "Ground reaction force after a sideways push as a measure of balance in recovery from stroke," Clinical Rehabilitation, vol. 14, no. 1, pp. 88-95, 2000.

[31] S. E. Lamb, L. Ferrucci, S. Volapto, L. P. Fried, and J. M. Guralnik, "Risk factors for falling in home-dwelling older women with stroke: the women's health and aging study," Stroke, vol. 34, no. 2, pp. 494-500, 2003.

[32] R. Teasell, M. McRae, N. Foley, and A. Bhardwaj, "The incidence and consequences of falls in stroke patients during inpatient rehabilitation: factors associated with high risk," Archives of Physical Medicine and Rehabilitation, vol. 83, no. 3, pp. 329-333, 2002.

[33] R. W. Bohannon, "Gait performance of hemiparetic stroke patients: selected variables," Archives of Physical Medicine and Rehabilitation, vol. 68, no. 11, pp. 777-781, 1987.

[34] P. J. Friedman, "Gait recovery after hemiplegic stroke," International Disability Studies, vol. 12, no. 3, pp. 119-122, 1990.

[35] A. L. Hsu, P. F. Tang, and M. H. Jan, "Analysis of impairments influencing gait velocity and asymmetry of hemiplegic patients after mild to moderate stroke," Archives of Physical Medicine and Rehabilitation, vol. 84, no. 8, pp. 1185-1193, 2003.

[36] B. Kollen, G. Kwakkel, and E. Lindeman, "Hemiplegic gait after stroke: Is measurement of maximum speed required?" Archives of Physical Medicine and Rehabilitation, vol. 87, no. 3, pp. 358-363, 2006.

[37] S. R. Sheldon, I. A. Kramers de Quervain, S. Leurgans, W. S. Pease, and D. McAllister, "Gait pattern in the early recovery period after stroke," Journal of Bone and Joint Surgery, vol. 78, no. 10, pp. 1506-1514, 1996.

[38] A. L. Hall, C. L. Peterson, S. A. Kautz, and R. R. Neptune, "Relationships between muscle contributions to walking subtasks and functional walking status in persons with post-stroke hemiparesis," Clinical Biomechanics, vol. 26, no. 5, pp. 509515, 2011.

[39] C. P. McGowan, R. Kram, and R. R. Neptune, "Modulation of leg muscle function in response to altered demand for body support and forward propulsion during walking," Journal of Biomechanics, vol. 42, no. 7, pp. 850-856, 2009.

[40] K. K. Patterson, I. Parafianowicz, C. J. Danells et al., "Gait asymmetry in community-ambulating stroke survivors," Archives of Physical Medicine and Rehabilitation, vol. 89, no. 2, pp. 304-310, 2008.

[41] B. French, L. H. Thomas, M. J. Leathley et al., "Repetitive task training for improving functional ability after stroke," Stroke, vol. 40, no. 4, pp. e98-e99, 2009.

[42] L. Wevers, I. van de Port, M. Vermue, G. Mead, and G. Kwakkel, "Effects of task-oriented circuit class training on walking competency after stroke: a systematic review," Stroke, vol. 40, no. 7, pp. 2450-2459, 2009.

[43] A. Lamontagne, C. Paquette, and J. Fung, "Stroke affects the coordination of gaze and posture during preplanned turns while walking," Neurorehabilitation and Neural Repair, vol. 21, no. 1, pp. 62-67, 2007.

[44] T. Imai, S. T. Moore, T. Raphan, and B. Cohen, "Interaction of the body, head, and eyes during walking and turning," Experimental Brain Research, vol. 136, no. 1, pp. 1-18, 2001.

[45] D. Xu, L. G. Carlton, and K. S. Rosengren, "Anticipatory postural adjustments for altering direction during walking," Journal of Motor Behavior, vol. 36, no. 3, pp. 316-326, 2004.

[46] A. E. Patla, A. Adkin, and T. Ballard, "Online steering: coordination and control of body center of mass, head and body reorientation," Experimental Brain Research, vol. 129, no. 4, pp. 629-634, 1999.

[47] M. S. Orendurff, A. D. Segal, J. S. Berge, K. C. Flick, D. Spanier, and G. K. Klute, "The kinematics and kinetics of turning: limb asymmetries associated with walking a circular path," Gait \& Posture, vol. 23, no. 1, pp. 106-111, 2006.

[48] L. A. Simpson, W. C. Miller, and J. J. Eng, "Effect of stroke on fall rate, location and predictors: a prospective comparison of older adults with and without stroke," PLoS One, vol. 6, no. 4, Article ID e19431, 2011.

[49] D. Hyndman, A. Ashburn, and E. Stack, "Fall events among people with stroke living in the community: circumstances of falls and characteristics of fallers," Archives of Physical Medicine and Rehabilitation, vol. 83, no. 2, pp. 165-170, 2002.

[50] R. G. Cumming and R. J. Klineberg, "Fall frequency and characteristics and the risk of hip fractures," Journal of the American Geriatrics Society, vol. 42, no. 7, pp. 774-778, 1994.

[51] S. F. H. Mackintosh, K. Hill, K. J. Dodd, P. Goldie, and E. Culham, "Falls and injury prevention should be part of every stroke rehabilitation plan," Clinical Rehabilitation, vol. 19, no. 4, pp. 441-451, 2005.

[52] M. T. Thigpen, K. E. Light, G. L. Creel, and S. M. Flynn, "Turning difficulty characteristics of adults aged 65 years or older," Physical Therapy, vol. 80, no. 12, pp. 1174-1187, 2000.

[53] K. Hase and R. B. Stein, "Turning strategies during human walking," Journal of Neurophysiology, vol. 81, no. 6, pp. 29142922, 1999.

[54] M. J. D. Taylor, P. Dabnichki, and S. C. Strike, "A threedimensional biomechanical comparison between turning 
strategies during the stance phase of walking," Human Movement Science, vol. 24, no. 4, pp. 558-573, 2005.

[55] M. Roerdink, C. J. C. Lamoth, G. Kwakkel, P. C. W. van Wieringen, and P. J. Beek, "Gait coordination after stroke: Benefits of acoustically paced treadmill walking," Physical Therapy, vol. 87, no. 8, pp. 1009-1022, 2007.

[56] K. L. Hollands, M. A. Hollands, D. Zietz, A. Miles Wing, C. Wright, and P. van Vliet, "Kinematics of turning 180。 during the timed up and go in stroke survivors with and without falls history," Neurorehabilitation and Neural Repair, vol. 24, no. 4, pp. 358-367, 2010.

[57] M. Hong, J. S. Perlmutter, and G. M. Earhart, "A kinematic and electromyographic analysis of turning in people with Parkinson disease," Neurorehabilitation and Neural Repair, vol. 23, no. 2, pp. 166-176, 2009.

[58] K. Berg, S. Wood-Dauphinee, and J. I. Williams, "The balance scale: reliability assessment with elderly residents and patients with an acute stroke," Scandinavian Journal of Rehabilitation Medicine, vol. 27, no. 1, pp. 27-36, 1995.

[59] C. Faria, L. F. Teixeira-Salmela, and S. Nadeau, "Effects of the direction of turning on the timed up \& go test with stroke subjects," Topics in Stroke Rehabilitation, vol. 16, no. 3, pp. 196-206, 2009.

[60] M. E. Tinetti, T. Franklin Williams, and R. Mayewski, "Fall risk index for elderly patients based on number of chronic disabilities," American Journal of Medicine, vol. 80, no. 3, pp. 429-434, 1986.

[61] K. Berg, "Measuring balance in the elderly: preliminary development of an instrument," Physiotherapy Canada, vol. 41, no. 6, pp. 304-311, 1989.

[62] D. A. Winter, "Human balance and posture control during standing and walking," Gait \& Posture, vol. 3, no. 4, pp. 193214, 1995.

[63] A. D. Segal, M. S. Orendurff, J. M. Czerniecki, J. B. Shofer, and G. K. Klute, "Local dynamic stability in turning and straightline gait," Journal of Biomechanics, vol. 41, no. 7, pp. 14861493, 2008.

[64] S. C. Strike and M. J. D. Taylor, "The temporal-spatial and ground reaction impulses of turning gait: Is turning symmetrical?" Gait \& Posture, vol. 29, no. 4, pp. 597-602, 2009.

[65] M. Woollacott and A. Shumway-Cook, "Attention and the control of posture and gait: a review of an emerging area of research," Gait \& Posture, vol. 16, no. 1, pp. 1-14, 2002.

[66] H. Pashler, "Dual-task interference in simple tasks: data and theory," Psychological Bulletin, vol. 116, no. 2, pp. 220-244, 1994.

[67] L. Bensoussan, J. M. Viton, M. Schieppati et al., "Changes in postural control in hemiplegic patients after stroke performing a dual task," Archives of Physical Medicine and Rehabilitation, vol. 88, no. 8, pp. 1009-1015, 2007.

[68] D. Hyndman, R. M. Pickering, and A. Ashburn, "Reduced sway during dual task balance performance among people with stroke at 6 and 12 months after discharge from hospital," Neurorehabilitation and Neural Repair, vol. 23, no. 8, pp. 847854, 2009.

[69] S. O'Shea, M. E. Morris, and R. Iansek, "Dual task interference during gait in people with Parkinson disease: effects of motor versus cognitive secondary tasks," Physical Therapy, vol. 82, no. 9, pp. 888-897, 2002.

[70] Y. R. Yang, Y. C. Chen, C. S. Lee, S. J. Cheng, and R. Y. Wang, "Dual-task-related gait changes in individuals with stroke," Gait \& Posture, vol. 25, no. 2, pp. 185-190, 2007.
[71] I. Melzer, I. Tzedek, M. Or et al., "Speed of voluntary stepping in chronic stroke survivors under single- and dualtask conditions: a case-control study," Archives of Physical Medicine and Rehabilitation, vol. 90, no. 6, pp. 927-933, 2009.

[72] A. Bowen, R. Wenman, J. Mickelborough, J. Foster, E. Hill, and R. Tallis, "Dual-task effects of talking while walking on velocity and balance following a stroke," Age and Ageing, vol. 30, no. 4, pp. 319-323, 2001.

[73] P. Plummer-D'Amato, L. J. P. Altmann, D. Saracino, E. Fox, A. L. Behrman, and M. Marsiske, "Interactions between cognitive tasks and gait after stroke: a dual task study," Gait \& Posture, vol. 27, no. 4, pp. 683-688, 2008.

[74] I. Melzer, M. Goldring, Y. Melzer, E. Green, and I. Tzedek, "Voluntary stepping behavior under single- and dual-task conditions in chronic stroke survivors: a comparison between the involved and uninvolved legs," Journal of Electromyography and Kinesiology, vol. 20, no. 6, pp. 1082-1087, 2010. 


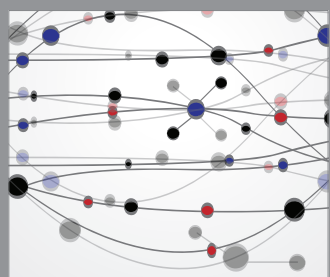

The Scientific World Journal
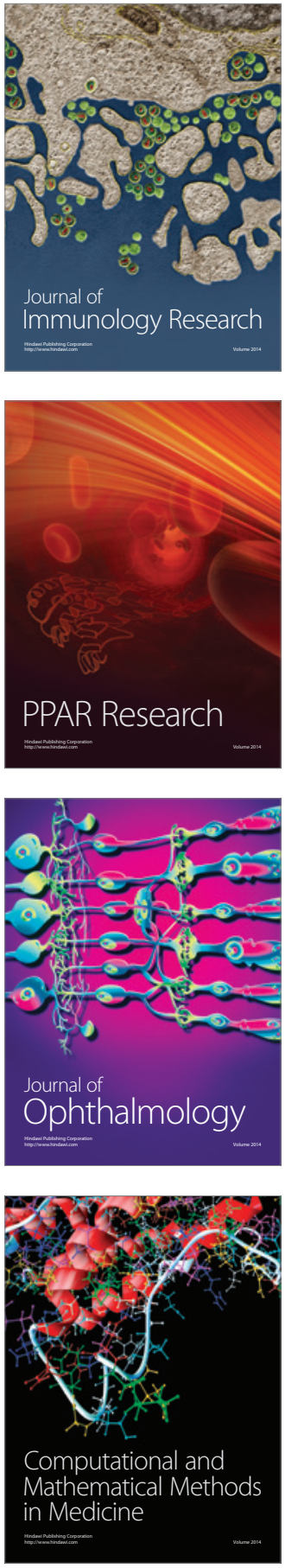

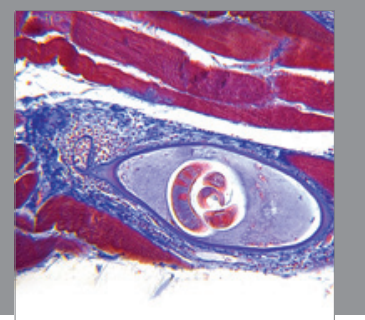

Gastroenterology

Research and Practice
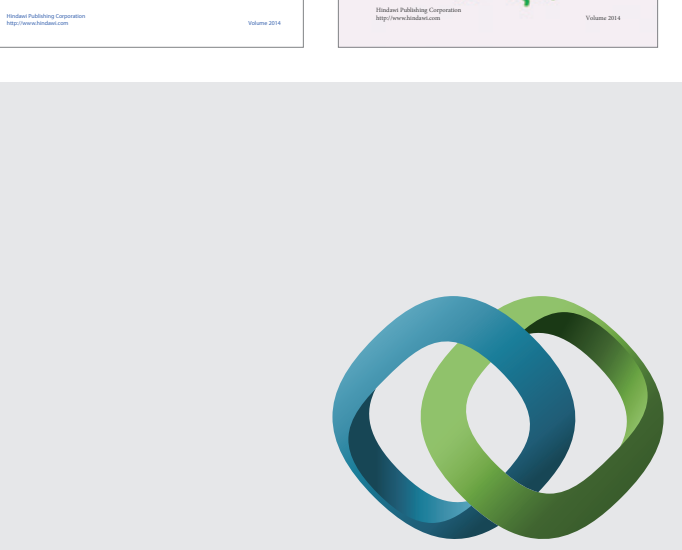

\section{Hindawi}

Submit your manuscripts at

http://www.hindawi.com
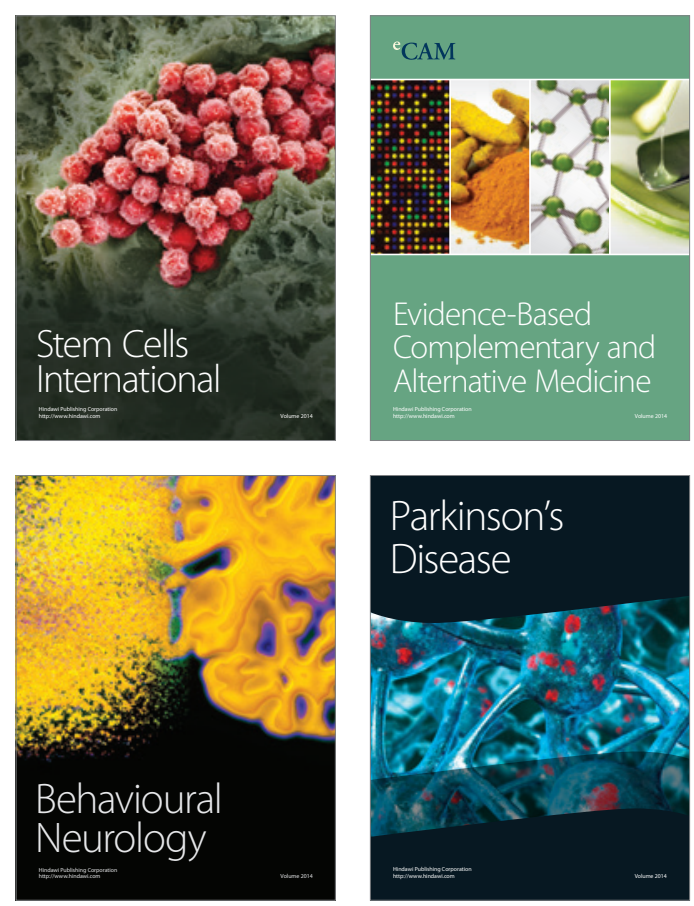

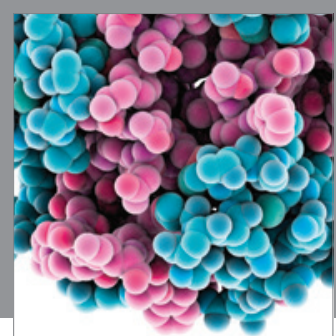

Journal of
Diabetes Research

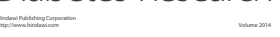

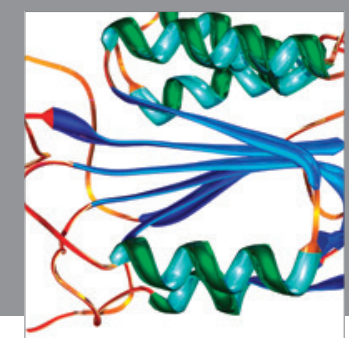

Disease Markers
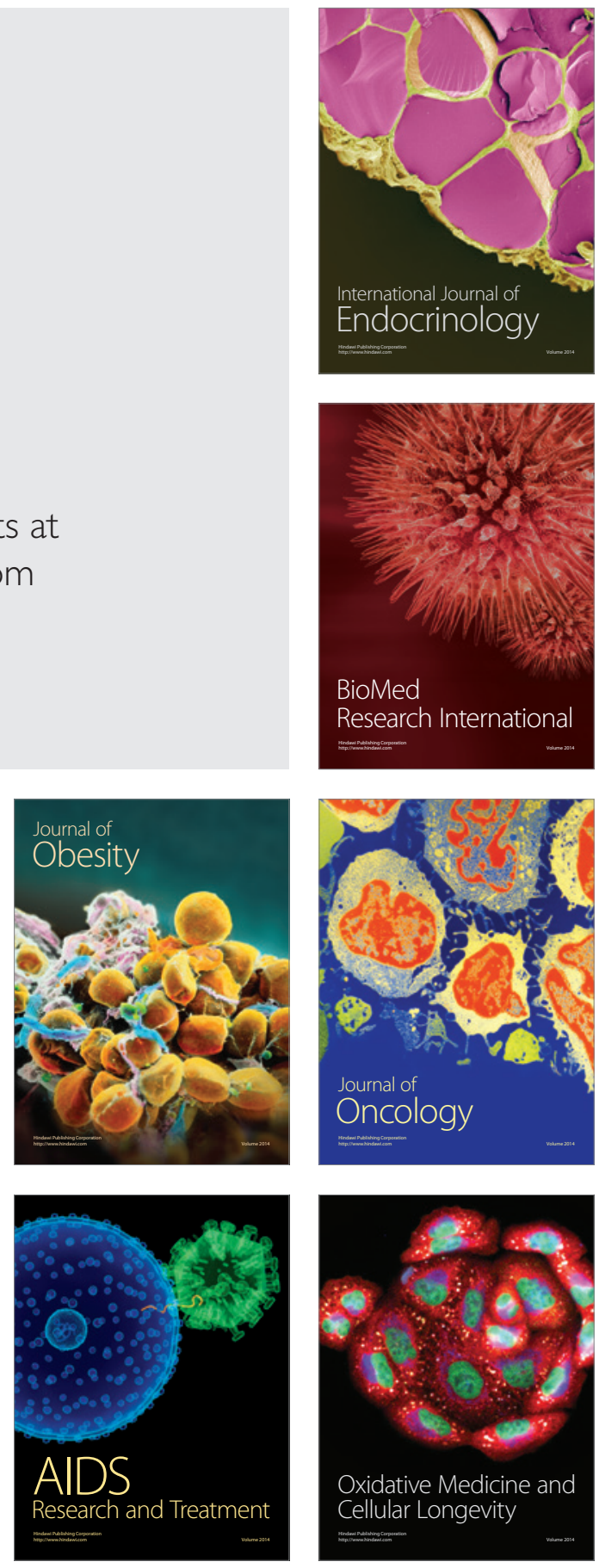